\title{
The research of international and national educational standards on leadership development for pharmacy students
}

\author{
Nataliia Aliekperova* \\ Dep. of Organisation and Economics of Pharmacy, Bogomolets National Medical University, Kyiv, Ukraine
}

\begin{abstract}
To estimate the level of usage of leadership development opportunities, International Pharmaceutical Federation official data and national educational and professional standards for pharmacy students in such countries as the USA, the United Kingdom, Australia, and Canada have been analyzed. According to the results, leadership knowledge and skills are of great importance to provide people with advanced pharmaceutical care. The most common leadership abilities for students getting pharmaceutical education deal with personal leadership (self-awareness, self-motivation, emotional intelligence, innovative mindset), teamwork, effective collaboration with other health professionals, building of a shared vision, resulting in the ability to become a change agent for sustainable development and improvement of health care system.
\end{abstract}

\section{Introduction}

The successful development of pharmaceutical organizations and the usage of leadership principles demand focusing attention on the study of the fundamental basis of leadership in the system of pharmaceutical education. As noted in Junk et al. (2016), "leadership is a professional obligation, and, to that end, orientation should emphasize leadership by all as essential to the future of the pharmacy profession, essential to developing and implementing new pharmacy services designed to improve patient outcomes, and essential to society" [1]. Pharmacy students should receive theoretical knowledge about leadership, leadership traits and skills, including such abilities as to work in a team efficiently, to support interprofessional collaboration, to use the features of corporate culture for goals achieving, to become an agent of change, etc. This leadership knowledge must be practically applied through exercising tasks and projects, including cooperation with peers, for the continuous improvement of leadership traits and skills, and the existing healthcare system.

The purpose of the study is the comprehensive analysis of leadership development opportunities for pharmacy students based on systematic literature review. Tasks of the research:

- to analyse the recommendations of the International Pharmaceutical Federation (FIP) as regards the importance of the development of leadership competencies for pharmacists;

- to study national educational and professional standards in pharmacy in such countries as the USA, the United Kingdom, Australia, Canada, as regards the development of leadership knowledge and skills;

*Corresponding author: aliekperova18@gmail.com
- to study key leadership competencies and leadership development opportunities for pharmacy students in highly developed western countries.

\section{Methods}

Firstly, it is necessary to conduct the topicality analysis of the aspects, dedicated to leadership and leadership competencies for pharmacy professionals based on official FIP materials. These documents are formed on outcomes of the global conference on pharmacy and pharmaceutical sciences education in Nanjing (China) in 2016 , and they are available free on the official site of FIP $[2,3,4]$.

The next step is the analysis of national educational and professional standards for pharmacists in the USA, the United Kingdom, Australia, and Canada. The choice of these countries was determined by certain reasons, namely, the accessibility of official materials in English and the high level of pharmaceutical education. Thus, according to QS world university rankings 2020 "pharmacy and pharmacology», - the top 10 universities are in these countries, furthermore, five leaders are presented by University of Oxford (the United Kingdom), Monash University (Australia), Harvard University (the United States), University of Toronto (Canada), University College London (the United Kingdom) [5]. What is more, the core leadership theories are invented by scientists from these countries, particularly from the USA (Ralph Stogdill, James Burns, Bernard Bass, James Kouzes, Barry Posner, Warren Bennis, Bert Nanus, Brus Avolio, Margaret Wheatley, Ron Heifetz, and many others).

Taking into account the keen focus on leadership issues in the USA, scientific publications in such 
American journals as the American Journal of Pharmaceutical Education and the Currents in Pharmacy Teaching and Learning have been analyzed. It allowed to highlight the main leadership development competencies (Junk et al. 2013, 2016) [1,6], as well as to study their importance for students on the basis of Delphi method, a survey of students and a systematic review of the related literature (Feller et al., 2016 and Tucci et al., 2017, Reed et al., 2019) [7, 8, 9]. The description of leadership development opportunities in the USA, as a rule, of courses and projects, the research of students' preferences regarding their content (Panther et al., 2017, Feller et al., 2016, Sucher et al., 2013, Patterson et al, 2013) might lead to the formation of key priorities on leadership issues for pharmacy students $[7,10,11,12]$.

\section{Results and discussion}

\section{Role of leadership global workforce development - International Pharmaceutical Federation (FIP)}

Nowadays, leadership problems in pharmaceutical area are of great importance. Thus, the global vision for education and workforce has been formulated by FIP in line with the outcomes of global conference on pharmacy and pharmaceutical sciences education, which took place in Nanjing (China) in November 2016. The vision underlines the core postulates of transformational and adaptive theories of leadership, namely the necessity to form "adaptable pharmaceutical workforces" and implement "collaboration with other stakeholders in health care" through "transformative and continuous education" for advanced health care [2].

According to the vision, FIP developed the main workforce development goals; one of them is the leadership development, in which an emphasis is placed on the upgrowth of leadership skills at all stages of career development, including education and science. On top of that, in such a workforce development goal as competency development attention is drawn to "clear policy that links leadership development (from early years) with competence attainment for the advancement of practice activities" [3].

On the grounds of Global Conference in Nanjing (China) outcomes, FIP published the Statements on Pharmacy and Pharmaceutical Sciences Education aimed at the improvement of the system of pharmaceutical education. These Statements consist of 8 clusters; and one of them is devoted to leadership - Foundation Training and Leadership. This cluster is focused on the need to include leadership development issues to the educational process for the formation of future pharmacy leaders, who can take full responsibility for the sustainable development and the improvement of the health care system [4]. Fullan (2003) names such leaders system thinkers and observes that they "...will gravitate toward strategies that alter people's system-related experience; that is, they will alter people's mental awareness of the system as whole, theryby contributing to altering the system itself" [13].
2. Pharmacist leadership development as an educational and professional obligation in the USA, the United Kingdom, Australia and Canada

\section{].1 USA}

\subsubsection{Leadership development outcomes for pharmacy students}

In the USA, a considerable transformation towards leadership popularization in pharmaceutical education has taken place [in the USA.] Thus, the Center for the Advancement of Pharmacy Education (CAPE) released the fourth version of educational outcomes for the justification of the curricular priorities of the Doctor of Pharmacy programs in 2013 [14]. The CAPE considerably enlarged students' professional knowledge and skills, making a special emphasis on self-awareness, innovation, leadership and professionalism. Besides, such abilities as progressive collaboration of pharmacists in a team, including interprofessional collaboration, providing pharmaceutical care for diverse patients, contributing to the health and well-being both of individuals and communities as a whole, consistent and continuous improvement of professional knowledge and skills, as well the ability to do well in a highly technical workplace are highlighted [14].

In accordance to educational outcome 4.2 - leadership - future pharmacy professionals (PharmD) must "demonstrate responsibility for creating and achieving shared goals, regardless of position", as well as understand the essence of leadership, differences between leadership and management, features of working in a team for getting sustainable and progressive results. However, proposed learning objectives are only samples that need to be enlarged and specified into definite leadership competencies. What is more, Junk et al. (2016) paid attention that other CAPE-2013 educational outcomes (patient advocacy, interprofessional collaboration, communication, professionalism, innovation and entrepreneurship and others) are connected to leadership competencies [1].

The Accreditation Council for Pharmacy Education (ACPE) released the Accreditation Standards and Key Elements for the Professional Program in Pharmacy Leading to the Doctor of Pharmacy Degree ("Standards 2016") in 2016, which fully reflect the CAPE-2013 educational outcomes [15]. Moreover, didactic content areas are enlarged in comparison with the previous the ACPE standard, namely Professional Development is added to Social and Behavioral Aspects of Practice. It is worth saying, that these amendments illustrate the fourth domain of educational outcomes of the CAPE - 2013 Personal and Professional Development (self-awareness, innovation, leadership and professionalism).

\subsubsection{Leadership competencies of pharmacy students}

The appropriate leadership competencies for pharmacy students should be developed to implement educational 
outcomes in practice. These competencies make the basis for the formation of pharmacist leadership development opportunities. So, Junk et al. (2013) in the comprehensive expert survey of twenty-six leadership instructors, proposed 11 leadership competencies for pharmacy students, which are divided into three parts:

- leadership knowledge - 4 competencies (explain the importance of leadership in pharmacy, recognize that leadership comes from those with and without titles, distinguish between leadership and management, describe the characteristics, behaviors and practices of effective leaders);

- personal leadership commitment - 2 competencies (demonstrate self-awareness in leadership, engage in personal leadership development);

- leadership skill development - 5 competencies (develop a shared vision for an initiative or project, collaborate with others, lead members of a team, develop knowledge of organizational culture, outline change processes) [6].

Feller et al. (2016) surveyed pharmacy program faculty members and staff employed by the pharmacy program as to how their leadership development opportunities correspond to 11 leadership development competencies [7]. Thus, such competencies as "Collaborate with others" and "Describe the characteristics, behaviors, and practices of effective leaders" received the highest indices, 9.3 out of 10 and 8.6 out of 10 , respectively. Leadership competencies with the lowest indices are "Develop knowledge of organizational culture"- 5.3 and "Distinguish between leadership and management" - 6.5. Tucci et al. (2017) analyzed the answers of 362 students getting pharmaceutical education for the assessment of their perceptions and self-efficacy in leadership competencies, offered by Junk et al. (2013) [6, 8]. The research indicates that respondents, actively engaged in pharmacy organizations leadership, for instance, by having an eboard position or service as the chair of a committee, demonstrate better results compared to other students. Generally, their higher estimation of their own efficiency concerned their leadership knowledge and personal leadership commitment. The results of the study are confirmed by the words of Posner (2009) who remarks that "leadership requires doing and leadership development therefore requires action-learning (or learning on the job) to find one's voice, develop, and hone one's skills" [16].

Leadership competencies for future pharmacists as proposed by Junk et al. (2013) are just recommended, and they can be changed and adjusted in line with the vision of a definite university or faculty [6]. According to Junk et al. (2016), the guiding principles that were developed through the Delphi process with participation of twenty-six leadership instructors, must be the basis of leadership competencies [1]. The leadership competencies must include self-focused leadership competencies, knowledge-based competencies, behavior-based competencies, as well as the cognitive domain and the affective domain. In fact, leadership competencies might be reflected in the definition of authentic leadership, suggested by Griffith et al. (2009): "a pattern of leader behavior that draws upon and promotes both positive psychological capacities and a positive ethical climate, to foster greater self-awareness, an internalized moral perspective, balanced processing of information, and relational transparency on the part of leaders working with followers, fostering positive selfdevelopment" [16]. Beside authentic leadership, such leadership theories as transformational and adaptive theories can be the framework of leadership competencies, even though they focus more on the relations between the leader and the followers, which already implies leadership authenticity. Thus, among 11 leadership competencies only two can be related to transformational and adaptive leadership - to develop a shared vision and to lead members of a team.

\subsubsection{Leadership development opportunities for pharmacy students}

According to the results, 94 schools of pharmacy out of 138 propose leadership development opportunities for students getting pharmaceutical education. Overall, 191 student pharmacist leadership development opportunities have been identified, most of them are courses $(41.9 \%)$, followed by projects/programs and series of events ( $18.8 \%$ and $10.9 \%$ respectively). Generally, they are didactic $(83.6 \%)$ and almost $30 \%$ are compulsory for all pharmacy students [7]. In addition, Feller et al. (2016) gave a brief description of some leadership development opportunities, offered by Regis University ReuckertHartman College for Health Professions, Palm Beach Atlantic University Lloyd L. Gregory School of Pharmacy, University of Minnesota College of Pharmacy, Concordia University College of Pharmacy, etc. [7].

Some schools of pharmacy have not only created leadership development opportunities and have begun to implement them in the educational process, also they have analyzed their efficiency. For instance, the elective course dedicated to leadership development at Regis University Reuckert-Hartman College for Health Professions (Sucher et al., 2013) has not been only characterized in the context of learning outcomes, topics and main educational tools, but in terms of its efficiency and practical usefulness for students getting the PharmD degree [11]. Thus, topics devoted to the research of one's own leadership authenticity, one's own strengths, including such assignments as StrengthsFinder, Strength Deployment Inventory, Kolb Learning Style Inventory, and others were defined by students as the most valuable ones. According to the survey results, more than $90 \%$ of students who passed through this elective course, 'strongly agree' that "this course helped me learn more about aspects of my character that are essential for leaders". Leadership development opportunities at The University of Iowa College of Pharmacy, (Patterson et al, 2013) [12], at University of Washington и Washington State University College of Pharmacy (Panther et al, 2017) also confirmed their effectiveness 
in "developing leadership skills in students and inspiring student pharmacists to be agents of change" [10].

Despite the application of leadership opportunities in many schools of pharmacy in the USA, at present, the unified definition of leadership in pharmacy is absent, leadership competencies vary considerably, operationalization of main concepts and terms needs development (Reed et al., 2019) [9]. What is more, fullfledged and sustainable student leadership development in pharmacy depends not only on the existence of a suitable course or project, but on the creation of innovative and supportive climate at an educational institution (Junk et al, 2016) [1]. Bradley-Baker et al., 2016 notes that "colleges and schools should strive to create an environment where leadership is not only recognized but valued" and "creating a supportive network of role models and mentors (faculty members, peers, and practitioners) is important to professional growth" [18].

\subsection{The United Kingdom}

\subsubsection{Leadership development outcomes and competencies of pharmacy students}

The General Pharmaceutical Council is a regulatory authority for pharmacists, pharmacy technicians and registered pharmacies in the United Kingdom that released the Standards for Pharmacy Professionals in 2017. According to them, pharmacy professionals must demonstrate leadership [19].

The Royal Pharmaceutical Society, the professional membership body for pharmacists and pharmacy in the United Kingdom, published the Professional Standards for Public Health Practice for Pharmacy in 2014 [20]. It contains 9 main standards directed on the provision of "a framework to support pharmacists and their teams to improve public health services, and shape future services and pharmacy roles to deliver quality patient care and improve health outcomes". In Standard 8.0 "Strategic Leadership and Collaborative Working for Health" attention is paid to the formation of leadership on three levels, namely national and local governments, teams of pharmacists and individual pharmacists. Also, actual examples are provided of the main aspects of the standard implementation in community pharmacy, hospital pharmacy and primary care, and local authorities. The standard devoted to leadership underlines the necessity of pharmacy leaders, teams, and pharmacists to collaborate with each other and other health professionals effectively to improve the population's health.

In more detail, leadership in pharmacy is considered in the Leadership Development Framework released by the Royal Pharmaceutical Society in 2015 [21]. Actually, this document reflects the NHS Leadership Academy's Healthcare Leadership Model, at the same time it focuses on pharmacists and pharmaceutical scientists [22]. The Leadership Development Framework "emphasizes the collective responsibility of everyone in pharmacy to seek to contribute to the leadership process and to develop and empower the leadership capacity of colleagues". It consists of 9 domains (inspiring shared purpose, leading with care, evaluating information, connecting our service, sharing the vision, engaging the team, holding to account, developing capability, influencing for results), each of them describes the recommends pharmacists' behavior on a four-part scale: essential, proficient, strong and exemplary [21].

\subsubsection{Leadership development opportunities of pharmacists}

Leadership development opportunities are proposed as a postgraduate education in the United Kingdom. The Centre for Pharmacy Postgraduate Education (CPPE), that is a part of the Division of Pharmacy and Optometry at the University of Manchester, carries out leadership trainings for pharmacists and pharmacy technicians in line with the Royal Pharmaceutical Society's Leadership Development Framework. For example, the CPPE leadership school offers a four-day program directed on the development of leadership knowledge and skills, such as self-awareness, estimation of own strengths, values, preferred ways of working, leadership styles. Also, the training aims at the development of such abilities as a team-leading, conflict and change management in organizations to improve pharmaceutical care and proactive healthy lifestyle advice [23].

\section{૧ [3 Australia}

The Pharmaceutical Society of Australia released a new National Competency Standards Framework for Pharmacists in Australia in 2016, which presents the description of advanced competencies for a pharmacist to "meet medication and related service needs, so that both optimal health outcomes and economic objectives are achieved for Australians" [24]. According to this standard, competencies must be evaluated and improved consistently, starting from self-management and own professional activities, then cooperation with professional peers and colleagues, followed by an orientation on a teamwork and leading team-based activities and, finally focus on the integration of various functions and processes for the implementation of positive changes in the future. In real fact, the fullfledged realization of this concept requires the sustainable development of leadership knowledge and skills. It is worth noting, that those who are "working on the real problem of transforming real system and learning by doing it" are characterized by Fullan as "new theoreticians' [13].

The leadership development as an advanced competency is paid attention to in Domain 4 Leadership and management: standard 4.1 (show leadership of self) and standard 4.3 (show leadership in practice). Having been presented in the previous version of the National Competency Standards Framework for Pharmacists, Standard 4.1 focuses on emotional awareness, self-regulation, self-assessment, selfmotivation and innovative mindset. However, Standard 
4.3 was introduced only in a new version in 2016; in it competencies are considered in the context of cooperation and a team-leading, formation of shared vision, supporting and coaching other people, as well as facilitating innovations and positive changes. The suggested in the document enabling competencies are characterized thoroughly not only at a general level with evidence examples, but in the context of advanced practice at three stages: transition level (stage 1), consolidation level (stage 2) and advanced level (stage 3). The achievement of competencies at these stages means both the incremental level of leadership knowledge and skills, and the application of efforts to go beyond the "comfort zone" [24].

\section{] .4 Canada}

The Canadian Council for Accreditation of Pharmacy Programs (CCAPP) developed the Accreditation Standards for Canadian First Professional Degree in Pharmacy Programs - 2018, where leadership skills for students getting pharmaceutical education are paid attention to [25]. Thus, Standard 4 (Criterion 4.3) proposes outcomes and competencies aimed at the development of leadership abilities for graduates. According to the Professional Competencies for Canadian at Entry to Practice Pharmacists prepared by National Association of Pharmacy Regulatory Authorities (NAPRA) in 2014, pharmacists and pharmacy technicians must "take responsibility for their decisions and provide leadership as appropriate", also, in line with the professional competency 8.2.2 they must "demonstrate leadership abilities in team processes" [26].

\section{Conclusions}

According to the results of literature review, official FIP materials, national educational and professional standards in such countries as the USA, the United Kingdom, Australia and Canada, it was found out that over the last time an emphasis has been put on leadership issues for pharmacy students. As a rule, the justifiability of leadership knowledge and skills development for pharmacy students is directly connected with the need to provide high-quality pharmaceutical care to the population.

Thereby, current educational programs and opportunities for students getting pharmaceutical education should pay attention to the development of such leadership skills as emotional intelligence, selfawareness, innovative mindset, teamwork, and collaboration with other healthcare professionals like physicians and nurses. What is more, future pharmaceutical workers should understand the general principles of organizational development for the successful management and sustainable transformation of pharmacies and the pharmaceutical industry. All in all, future pharmacists should become change agents for constant improvement of the health care system. The USA remains the most progressive country regarding the implementation of leadership development opportunities for students, considering the number of schools of pharmacy which propose various courses and projects devoted to leadership. At the same time, there are some discrepancies in leadership definition, knowledge and skills in pharmacy that requires using more unified approaches both in international and national educational standards.

\section{References}

1. K.K. Janke, M.H. Nelson, A.S. Bzowyckyj, D.G. Fuentes, E. Rosenberg E, and R. DiCenzo, Am J Pharm Educ, vol. 80(1):2, (2016),

2. International Pharmaceutical Federation (FIP), "Global Vision for Education and Workforce," (2016)

3. International Pharmaceutical Federation (FIP), "Pharmaceutical Workforce Development Goals," (2016)

4. International Pharmaceutical Federation (FIP), "Statements on Pharmacy and Pharmaceutical Sciences Education," (2017).

5. QS Top Universities, "University ranking: Pharmacy and Pharmacology," (2020)

6. K.K. Janke, A.P. Traynor, and C.J. Boyle, Am J Pharm Educ, 77(10):222, (2013),

7. T.T. Feller, W.R. Doucette, and M.J. Witry, Am J Pharm Educ, 80(5):79 (2016),

8. M. Tucci, K. Tong, K. Chia, and M. DiVall, Am J Pharm Educ, 83(2):6535, (2019),

9. B.N. Reed, A.M. Klutts, and T.J. Mattingly, Am J Pharm Educ, 83(9):7520 (2019)

10. S.G. Panther, R.A. Allen, K. Brantner, C.G. Jefferson, N.L. Murphy, and J.D. Robinson, Am J Pharm Educ, 83(5):6764, 2019,

11. B. Sucher, M. Nelson, and D. Brown Am J Pharm Educ, 77(10):224, (2013).

12. Accreditation Council for Pharmacy Education (ACPE), "Accreditation Standards and Key Elements for the Professional Program in Pharmacy Leading to the Doctor of Pharmacy Degree ("Standards 2016"),", Chicago, Illinois, (2016)

13. B.Z. Posner, Journal of Leadership Education, 8.1: 1-10 (2009).

14. J. Griffith, B. Avolio, T. Wernsing, and F.O. Walumbwa "What is authentic leadership development?" In book: Handbook of Positive Psychology and Work, Oxford, (2009)

15. L.R. Bradley-Baker and N.L. Murphy, "Leadership development of student pharmacists," Am J Pharm Educ, 77(10):219, (2013), doi:10.5688/ajpe7710219.

16. General Pharmaceutical Council, "Standards for pharmacy professionals," (2017)

17. Royal Pharmaceutical Society, "Professional Standards for Public Health Practice for Pharmacy," (2014) 
18. Royal Pharmaceutical Society, "Leadership Development Framework: Developing leadership where you are," (2015)

19. NHS Leadership Academy, "The Healthcare Leadership Model, version 1.0, Leeds: NHS Leadership Academy,"(2013)

20. Centre for Pharmacy Postgraduate Education, School of Pharmacy and Pharmaceutical Science, University of Manchester, "Inspiring Future Pharmacy Leaders: Learning portfolio," (2012)

21. Pharmaceutical Society of Australia. "National Competency Standards Framework for Pharmacists in Australia," (2016)

22. Canadian Council for Accreditation of Pharmacy Programs (CCAPP), “Accreditation Standards for
Canadian First Professional Degree in Pharmacy Programs," (2018)

23. National Association of Pharmacy Regulatory Authorities (NAPRA), "Professional Competencies for Canadian Pharmacists at Entry to Practice," (2014)

24. B.J. Patterson, O.W. Garza, M.J. Witry, E.H. Chang, D.E. Letendre, and C.B Trewet, Am J Pharm Educ, 77(10):223, (2013)

25. M. Fullan, Leadership \& Sustainability: System Thinkers in Action, Corwin Press, (2005)

26. M.S. Medina, C.M. Plaza, C.D. Am J Pharm Educ, 77(8):162 (2013) 